\title{
Polymer-Stabilized Sialylated Nanoparticles: Synthesis, Optimization, and Differential Binding to Influenza Hemagglutinins
}

\author{
Sarah-Jane Richards, Alexander N. Baker, Marc Walker, and Matthew I. Gibson*
}

Cite This: Biomacromolecules 2020, 21, 1604-1612

Read Online

ABSTRACT: During influenza infection, hemagglutinins (HAs) on the viral surface bind to sialic acids on the host cell's surface. While all HAs bind sialic acids, human influenza targets terminal $\alpha 2,6$ sialic acids and avian influenza targets $\alpha 2,3$ sialic acids. For interspecies transmission (zoonosis), HA must mutate to adapt to these differences. Here, multivalent gold nanoparticles bearing either $\alpha 2,6$ - or $\alpha 2,3$-sialyllactosamine have been developed to interrogate a panel of HAs from pathogenic human, low pathogenic avian, and other species' influenza. This method exploits the benefits of multivalent glycan presentation compared to monovalent presentation to increase affinity and investigate how multivalency affects selectivity. Using a library-orientated approach, parameters including polymer coating and core diameter were optimized for maximal binding and specificity were probed using galactosylated particles and a panel of biophysical techniques [ultraviolet-visible spectroscopy,

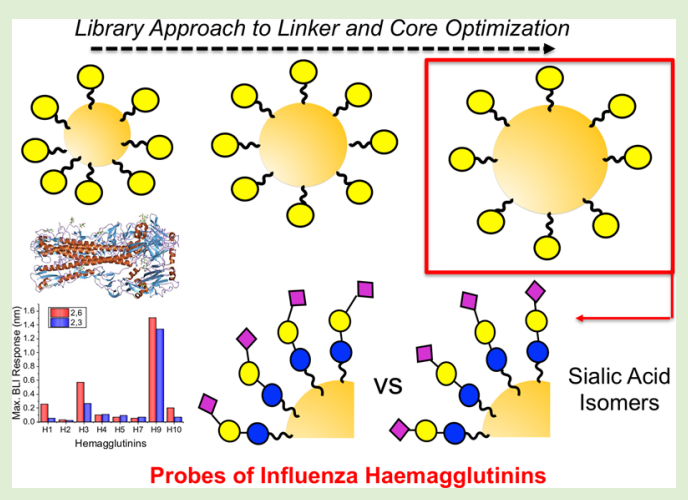
dynamic light scattering, and biolayer interferometry]. The optimized particles were then functionalized with sialyllactosamine and their binding analyzed against a panel of HAs derived from pathogenic influenza strains including low pathogenic avian strains. This showed significant specificity crossover, which is not observed in monovalent formats, with binding of avian HAs to human sialic acids and vice versa in agreement with alternate assay formats. These results demonstrate that precise multivalent presentation is essential to dissect the interactions of HAs and may aid the discovery of tools for disease and zoonosis transmission.

\section{INTRODUCTION}

Worldwide, annual epidemics of seasonal influenza are estimated to result in 3-5 million cases of severe illness, and up to 650,000 deaths annually are associated with respiratory diseases from seasonal influenza. ${ }^{1}$ While influenza A and B viruses cause seasonal epidemics, only A types have been evidenced to cause pandemics. Influenza $A$ viruses are classified into subtypes according to the combination of proteins on their surface; hemagglutinins (HA) and neuraminidases (NA)-for example, the $\mathrm{H} 1 \mathrm{~N} 1$ strain pandemic in 2009. ${ }^{2}$ During infection, influenza virus particles display trimeric HAs that bind to sialosides on the surface of their host cells to facilitate internalization. While all influenza strains bind sialosides, human strains preferentially bind to terminal $\alpha 2,6$ sialic acids, whereas avian influenza viruses show preference for $\alpha 2,3$ sialic acids. ${ }^{3,4}$ Zoonosis (interspecies transmission) can occur due to mutations in the HA, enabling a switch from $\alpha 2,3$ to $\alpha 2,6$. This allows avian influenza to infect humans, and often occurs in porcine (pig) hosts, which present both $\alpha 2,3$ to $\alpha 2,6$ sialosides in their respiratory tracts. ${ }^{5-7}$ Humans can be infected with avian, porcine, and other zoonotic influenza viruses including avian influenza subtypes; $\mathrm{H} 5 \mathrm{~N} 1, \mathrm{H} 7 \mathrm{~N} 9$, and H9N2 and swine flu virus subtypes; H1N1 (swine flu pandemic 2009), H1N2, and H3N2. ${ }^{8}$ It has also emerged that $\mathrm{N}$-glycolyl modifications (in addition to $\mathrm{N}$ - acetyl) play a significant role in species-dependent binding 9 and that influenza can bind phosphorylated, non-sialylated glycans determined against an array of $N$-linked glycans from human lungs, ${ }^{10}$ suggesting that other glycans are also involved, or potential targets during infection. ${ }^{11}$ Therefore, the study of HA interactions with sialic acids is essential to increase our understanding of this global pathogen and to help guide the discovery of new diagnostics and treatments.

The binding affinity of a HA toward an individual sialoside is weak $\left(K_{\mathrm{d}} \approx 2 \mathrm{mM}\right){ }^{12}$ but because of the multivalent presentation of both the HA ( 200-1000 copies/virus) and the dense layer of sialic acids on the cell surface, ${ }^{13}$ the affinity is significantly increased by the cluster glycoside effect. ${ }^{14}$ Hence, in vivo, HAs engage with cell-surface sialic acids which are not presented as free molecules in solution, but rather multivalent ensembles as in the glycocalyx. Strategies to probe and dissect HA function should reproduce this multivalent environment to

Received: February 6, 2020

Revised: March 19, 2020

Published: March 19, 2020 
be truly predictive. There are many examples where multivalent materials, in particular polymers, have been used to engage with carbohydrate binding proteins, with most studies focusing on plant lectins, which are of limited biomedical relevance. $^{15-19}$

Whitesides and co-workers synthesized sialic acid containing glycopolymeric inhibitors of influenza virus X-31 via a poly $(\mathrm{N}$ acrylyloxy succinimide) reactive scaffold. Using hemagglutination and enzyme-linked immunosorbent assays, the inhibitory activity of these polymers were found to be between $10^{3}$ and $10^{6}$ times greater than the free glycan. ${ }^{20,21}$ However, the effect of the linkage was not examined. Miura and co-workers synthesized 2,3- and 2,6-sialyllactose-functional glycopolymers that were able to recognize two different influenza strains. They found that 2,6-sialyllactose-functional glycopolymers inhibited hemagglutination caused by H3N2 (A/Aichi/2/68) but not H1N1 (A/Puerto Rico/8/34); conversely, 2,3sialyllactose-functional glycopolymers inhibited aggregation caused by the H1N1 strain but not the H3N2. ${ }^{22}$ The density and polymer length were found to affect the interaction with the virus. Trivalent presentation of 2,3 sialic acids was sufficient to give $>400$ fold increase in inhibitory activity against $\mathrm{H} 3$ influenzas. ${ }^{23}$ Godula and co-workers developed a microarray platform for the evaluation of virus binding based on 2,6- and 2,3-sialyllactose glycopolymers, which showed discrimination between different strains, with H3N2 (A/Aichi/ $2 / 68$ ) binding exclusively to 2,6-siallylactose glycopolymers, whereas H1N1 (A/Puerto Rico/8/34) bound to both 2,3- and 2,6 -sialyllactose polymers. ${ }^{24}$ In addition to the nature of the sialic acid, the 3-D presentation is a crucial determinant with bivalent ligands showing wide affinity trends based only on separation, $^{25}$ for example, H3N2 strains have evolved specificity related to the length of the sialosides linker. ${ }^{26}$ Furthermore, linear sialic acid polymers were found to be more potent inhibitors than dendritic. ${ }^{27}$

Gibson and co-workers have previously developed multivalent gold/polymer hybrids to dissect glycan binding interactions. ${ }^{28-31}$ Reversible addition fragmentation chain transfer (RAFT) polymerization was used to produce telechelic polymers that have a gold immobilization site at one end (masked thiol) and a glycan installation site (an amine reactive pentafluorophenyl group) at the other end. The polymer linker was found to be important, ensuring a stable formulation and fast response readout. ${ }^{28,30}$ The use of gold nanoparticle cores, exploiting their unique aggregation-induced red-blue color shift, provides label-free readouts. This has been used for the discrimination of human (H3N2) and avian (H5N1) influenza using PEGylated gold particles presenting trivalent $\alpha 2,6$ sialic acid ligands. ${ }^{32}$ Fairbanks and co-workers investigated the interaction of HAs and whole viruses with gold nanoparticles decorated with biantennary sialyloligosaccharides $(\alpha 2,6$ linked) extracted from egg yolk. Selectivity was observed between two H1N1 strains, A/New Caledonia/20/1999 and A/Puerto Rico/8/34, which have specificity toward $\alpha 2,6$ sialic acid and $\alpha 2,3$ sialic acid, respectively. ${ }^{33}$ De Geest and coworkers immobilized $\alpha 2,6$ sialic acid pendant glycopolymers on AuNPs and observed an interaction with inactivated H1N1 (A/Puerto Rico/8/34) viruses as determined by DLS; however, the selectivity compared with other strains was not investigated. ${ }^{34}$ Furthermore, Papp et al. showed the importance of gold core size on the efficiency of virus inhibitions with 14 $\mathrm{nm}$ particles having higher binding affinity than $2 \mathrm{~nm}$ particles. $^{35}$ The gold particle size enables the generation of large signal responses as well as multivalency; therefore, it is essential to optimize the core size, linker, and glycan. ${ }^{36}$ Lipid bilayers and dendrimer scaffolds have also been employed to probe or inhibit hemagglutinin binding..$^{37,38}$

Considering the above, the aim of this work was to develop a multivalent nanoparticle platform to dissect the binding trends of influenza HAs toward multivalent sialic acid isomers. A library-oriented screen of 27 nanoparticle formulations was used to identify the optimum size and linker length, and then translated to enable 2,3- and 2,6-sialyllactosamine attachment to multivalent gold nanoparticles. The binding affinities of these particles were evaluated by colorimetric aggregation assays, dynamic light scattering (DLS) and biolayer interferometry (BLI) to ensure the whole binding landscape was probed. A panel of eight HAs, including human and avian, were interrogated with the particles, and it was demonstrated that the multivalent presentation enables significant crossbinding between human and avian strains.

\section{EXPERIMENTAL SECTION}

Materials. All chemicals were used as supplied unless otherwise stated. N-Hydroxyethyl acrylamide (HEA) (97\%), 4,4'-azobis(4cyanovaleric acid) (ACVA) (98\%), mesitylene (reagent grade), triethylamine $(>99 \%)$, sodium citrate tribasic dihydrate $(>99 \%)$, gold(III) chloride trihydrate (99.9\%), and ammonium carbonate (reagent grade) were all purchased from Sigma-Aldrich. D(+)Galactosamine hydrochloride (99\%) was purchased from Acros Organics. 3'-Sialyllactose and 6'-sialyllactose were purchased from Carbosynth. 2-(Dodecylthiocarbonothioylthio)-2-methylpropionic acid pentafluorophenyl ester was synthesized as previously outlined by Richards and Gibson. ${ }^{28}$ Soybean agglutinin (SBA) and wheat germ agglutinin (WGA) were purchased from Vector Laboratories. The following reagents were obtained through BEI resources, NIAID, NIH: H1 HA protein from influenza virus, A/California/07/2009 (H1N1)pdm09, recombinant from baculovirus, NR-44074, H2 HA protein from influenza virus, A/Singapore/1/1957 (H2N2), recombinant from baculovirus, NR-2668, H3 HA protein from influenza virus, A/Uruguay/716/2007 (H3N2), recombinant from baculovirus, NR15168, H4 HA protein from influenza virus, $\mathrm{A} /$ mallard/Alberta/455/ 2015 (H4N6), recombinant from baculovirus, NR-51128, H5 HA protein from influenza virus, A/duck/Hunan/795/2002 (H5N1), recombinant from baculovirus, NR-43739, H7 HA protein from influenza virus, A/Canada/rv444/2004 (H7N3), recombinant from baculovirus, NR-43740, H9 HA protein from influenza virus, A/Hong Kong/33982/2009 (H9N2), recombinant from baculovirus, NR41792 and H10 HA protein with C-terminal histidine tag from influenza virus, A/harbor seal/Germany/1/2014 (H10N7), recombinant from baculovirus, NR-50172. Clear and black half are 96-well plates that were purchased from Greiner Bio-One. Streptavidin (SA) biosensors were purchased from ForteBio. Lectins and HAs were biotinylated using EZ-Link sulfo-NHS-LC-biotin reagent from Thermo Fisher Scientific using standard procedure (20-fold molar excess of biotin reagent, conjugation performed in phosphate-buffered saline (PBS) buffer and isolated using Amicon Ultra- $0.5 \mathrm{~mL} 3000$ MWCO centrifugal filters from Merck Millipore).

Physical and Analytical Methods. ${ }^{1} \mathrm{H}$ NMR spectra were recorded on Bruker DPX-300 and DPX-400 spectrometers using deuterated solvents purchased from Sigma-Aldrich. Chemical shifts are reported relative to residual non-deuterated solvent. Size exclusion chromatography (SEC) was carried out in dimethylformamide (DMF) and run on an Agilent 1260 Infinity II-MDS instrument equipped with differential refractive index, dual wavelength UV detectors, viscometry and light scattering detectors. The system was equipped with $2 \times$ PLgel mixed D columns $(300 \times 7.5 \mathrm{~mm})$ and a PLgel $5 \mu \mathrm{m}$ guard column. The eluent is DMF with $5 \mathrm{mM} \mathrm{NH}_{4} \mathrm{BF}_{4}$ additive. Samples were run at $1 \mathrm{~mL} \cdot \mathrm{min}^{-1}$ at $50{ }^{\circ} \mathrm{C}$. Poly(methyl methacrylate) (Agilent EasiVials) were used for calibration. Analyte 
Table 1. Polymers Prepared in This Study

$\begin{array}{ccc}\text { polymer } & {[\mathrm{M}] /[\mathrm{CTA}]^{a}} & \text { conversion }(\%)^{b} \\ \text { Gal-PHEA }_{10} & 10 & 96 \\ \text { Gal-PHEA }_{15} & 15 & 99 \\ \text { Gal-PHEA }_{20} & 20 & 97 \\ \text { Gal-PHEA }_{25} & 25 & 98 \\ \text { Gal-PHEA }_{30} & 30 & 95 \\ \text { Gal-PHEA }_{35} & 35 & 97 \\ \text { Gal-PHEA }_{40} & 40 & 98 \\ \text { Gal-PHEA }_{45} & 45 & 94 \\ \text { Gal-PHEA }_{50} & 50 & 92\end{array}$

$M_{\mathrm{n}(\mathrm{NMR})}\left(\mathrm{g} \cdot \mathrm{mol}^{-1}\right)^{b}$
1600
2200
2800
3600
3800
4600
5000
5700
5800

$M_{\mathrm{n}(\mathrm{SEC})}\left(\mathrm{g} \cdot \mathrm{mol}^{-1}\right)^{c}$
2600
3300
4200
5800
7700
9500
8900
9200
10,300

$M_{\mathrm{w}} / M_{\mathrm{n}}{ }^{\mathrm{c}}$
1.12
1.16
1.16
1.16
1.13
1.14
1.15
1.15
1.15

${ }^{a}$ Feed ratio of monomer to chain-transfer agent. ${ }^{b}$ Determined by ${ }^{1} \mathrm{H}$ NMR. ${ }^{c}$ Determined by SEC in dimethylformamide using poly (methyl methacrylate) standards. $M_{\mathrm{w}}$, weight average molecular weight; $M_{\mathrm{n}}$, number average molecular weight.

samples were filtered through a nylon filter with $0.22 \mu \mathrm{m}$ pore size before injection. Experimental molar mass $\left(M_{\mathrm{n}, \mathrm{SEC}}\right)$ and dispersity $(D)$ values of synthesized polymers were determined by conventional calibration using Agilent SEC/SEC software. The calibration range is 500-2,000,000 Da.

Lectin-Induced Aggregation Studies by Absorbance. A stock solution of lectin was made $\left(0.1 \mathrm{mg} \cdot \mathrm{mL}^{-1}\right.$ for SBA and $1 \mathrm{mg}$. $\mathrm{mL}^{-1}$ for WGA) in $10 \mathrm{mM}$ HEPES buffer with $0.15 \mathrm{M} \mathrm{NaCl}, 0.1 \mathrm{mM}$ $\mathrm{CaCl}_{2}$, and $0.01 \mathrm{mM} \mathrm{MnCl} 2$. A serial dilution $(25 \mu \mathrm{L})$ was made up in the same buffer in a clear, flat bottom, half area 96-well microtiter plate. GlycoAuNPs $(250 \mu \mathrm{L})$ were added to each well and incubated at room temperature for $30 \mathrm{~min}$. After $30 \mathrm{~min}$, an absorbance spectrum was recorded from 450 to $700 \mathrm{~nm}$ with $10 \mathrm{~nm}$ intervals.

Biolayer Interferometry. BLI was carried out on ForteBio Octet Red96 (ForteBio, USA). Assays were performed in black 96-well half area plates. Assays were carried out at $30^{\circ} \mathrm{C}$ and agitated at $1000 \mathrm{rpm}$. SA biosensor tips (ForteBio, USA) were hydrated in milliQ $\mathrm{H}_{2} \mathrm{O}$ water for at least $10 \mathrm{~min}$ prior to use. A stable baseline was established in milliQ water for $1 \mathrm{~min}$. The biosensors were functionalized by loading with $10 \mu \mathrm{g} / \mathrm{mL}$ biotinylated lectin or HA in PBS for $5 \mathrm{~min}$ followed by a $1 \mathrm{~min}$ equilibration step in $10 \mathrm{mM}$ HEPES with $0.15 \mathrm{M}$ $\mathrm{NaCl}$ and $0.1 \mathrm{mM} \mathrm{CaCl}$ and $\mathrm{MnCl}_{2}$ to remove any unbound protein and to establish a stable baseline. Following protein immobilization, the binding association with galactosylated and sialylated AuNPs was carried out in $10 \mathrm{mM}$ HEPES with $0.15 \mathrm{M} \mathrm{NaCl}$ and $0.1 \mathrm{mM} \mathrm{CaCl}_{2}$ and $\mathrm{MnCl}_{2}$ for 10 min followed by dissociation in $10 \mathrm{mM}$ HEPES with $0.15 \mathrm{M} \mathrm{NaCl}$ and $0.1 \mathrm{mM} \mathrm{CaCl}$ and $\mathrm{MnCl}_{2}$ for $10 \mathrm{~min}$.

Synthetic Section. Polymerization of Hydroxyethyl Acrylamide Using PFP-DMP. HEA (0.5 g, $4.34 \mathrm{mmol}$ ), pentafluorophenyl 2(dodecylthiocarbonothioylthio)-2-methylpropionic acid (PFP-DMP) (0.092 g, $0.17 \mathrm{mmol})$, and ACVA (0.0097 g, $0.034 \mathrm{mmol})$ were dissolved in 50:50 toluene/methanol $(4 \mathrm{~mL})$. Mesitylene $(150 \mu \mathrm{L})$ was added as an internal reference. An aliquot was taken for NMR analysis in $\mathrm{CDCl}_{3}$. The solution was degassed under $\mathrm{N}_{2}$ for $30 \mathrm{~min}$. The reaction was stirred at $70{ }^{\circ} \mathrm{C}$ for $90 \mathrm{~min}$. An aliquot was taken for NMR analysis in MeOD. The reaction was rapidly cooled in liquid nitrogen and precipitated into diethyl ether. The polymer was reprecipitated into diethyl ether from methanol twice to yield a yellow polymer product that was dried under vacuum. $96 \%$ conversion by NMR, $M_{\mathrm{n}}$ (theoretical $)=3400 \mathrm{~g} \cdot \mathrm{mol}^{-1} M_{\mathrm{n}}(\mathrm{SEC})=5800 \mathrm{~g} \cdot \mathrm{mol}^{-1}$ $M_{\mathrm{n}} / M_{\mathrm{w}}(\mathrm{SEC})=1.16$.

Gold Nanoparticle Synthesis. Gold nanoparticles of differing sizes were synthesized by the method developed by Bastús et al. ${ }^{39} \mathrm{~A}$ solution of $2.2 \mathrm{mM}$ sodium citrate in Milli-Q water $(150 \mathrm{~mL})$ was heated under reflux for $15 \mathrm{~min}$ under vigorous stirring. After boiling had commenced, $1 \mathrm{~mL}$ of $\mathrm{HAuCl}_{4}(25 \mathrm{mM})$ was injected. The color of the solution changed from yellow to bluish gray and then to soft pink in $10 \mathrm{~min}$. Immediately after the synthesis of the Au seeds and in the same reaction vessel, the reaction was cooled until the temperature of the solution reached $90{ }^{\circ} \mathrm{C}$. Then, $1 \mathrm{~mL}$ of $\mathrm{HAuCl}_{4}$ solution $(25 \mathrm{mM})$ was injected. After $30 \mathrm{~min}$, the reaction was finished. This process was repeated twice. After that, the sample was diluted by extracting $55 \mathrm{~mL}$ of the sample and adding $53 \mathrm{~mL}$ of MilliQ water and $2 \mathrm{~mL}$ of $60 \mathrm{mM}$ sodium citrate. This solution was then used as a seed solution, and the process was repeated a further 7 times. Generations 2, 5, and 8 were used for this study (Table 2).

Table 2. Nanoparticles Synthesized and Characterization

\begin{tabular}{cccccc} 
& & & & \multicolumn{2}{c}{ diameter $(\mathrm{nm})$} \\
\cline { 5 - 6 } particle & generation $^{a}$ & $\lambda_{\mathrm{SPR}}{ }^{b}$ & $A_{\mathrm{SPR}} / A_{450}{ }^{b}$ & $\mathrm{UV}^{\mathrm{N}}$ vis $^{c}$ & $\mathrm{DLS}^{d}$ \\
$\mathrm{Au}_{30}$ & 2 & 523 & 1.84 & 30 & $28.2 \pm 0.9$ \\
$\mathrm{Au}_{50}$ & 5 & 531 & 2.00 & 50 & $48.8 \pm 0.4$ \\
$\mathrm{Au}_{70}$ & 7 & 540 & 2.05 & 68 & $72.5 \pm 1.2$
\end{tabular}

${ }^{a}$ Generation of nanoparticles used from the seeding synthetic methodology (see Figure S1). ${ }^{b}$ Maximum absorption wavelength from the surface plasmon resonance band of the particles and characteristic ratio were used for diameter estimation. ${ }^{c}$ Estimated using the method of Haiss et al. ${ }^{41}$ from UV-vis. ${ }^{d}$ Diameter from DLS \pm standard error from three measurements.

\section{RESULTS AND DISCUSSION}

To access multivalent glycosylated gold nanoparticles, RAFT polymerization was employed to synthesize telechelic poly $(\mathrm{N}$ hydroxyethyl acrylamide) (PHEA) ligands suitable for both nanoparticle immobilization, and capture of glycans via a terminal pentafluoroethyl ester (PFP), ${ }^{28,30,31,40}$ (Figure 1A). A range of degrees of polymerization (DP) was targeted from DP10-DP50 by varying the $[\mathrm{M}] /[\mathrm{CTA}]$ ratio. The polymers were characterized by SEC confirming narrow dispersity (Figure 1C and Table 1). 2-Deoxy-2-amino galactosamine was conjugated to the $\omega$-terminal PFP, which also removes the dithiocarbonate to reveal a thiol for subsequent AuNP conjugation. ${ }^{19} \mathrm{~F}$ NMR (Figure 1D) was used to confirm displacement of the PFP group. Note, $\mathrm{GalNH}_{2}$ was chosen as the glycan to conduct initial screening, before progressing to the sialic acid particles (vide infra).

To access a range of gold nanoparticles of defined size, the kinetically controlled seeded growth strategy based on the Turkevich/Frens method via the reduction of $\mathrm{HAuCl}_{4}$ by sodium citrate was used. ${ }^{39}$ This involved a growth process leading to the enlargement of presynthesized $10 \mathrm{~nm}$ gold seeds via the surface-catalyzed reduction of $\mathrm{Au}^{3+}$ by sodium citrate (Figure 1B). This was characterized by UV-vis spectroscopy (Figure 1E), DLS (Figure 1F), and transmission electron microscopy (TEM) (Figure 1G-I). The three different nanoparticle sizes were coated with the nine different polymers to generate a diverse library of 27 multivalent glyconanoparticles, this convergent strategy maximized structural diversity. DLS measurements showed an increase in hydrodynamic diameter, along with a red-shift in the SPR peak $\left(\lambda_{\max }\right)$, both 

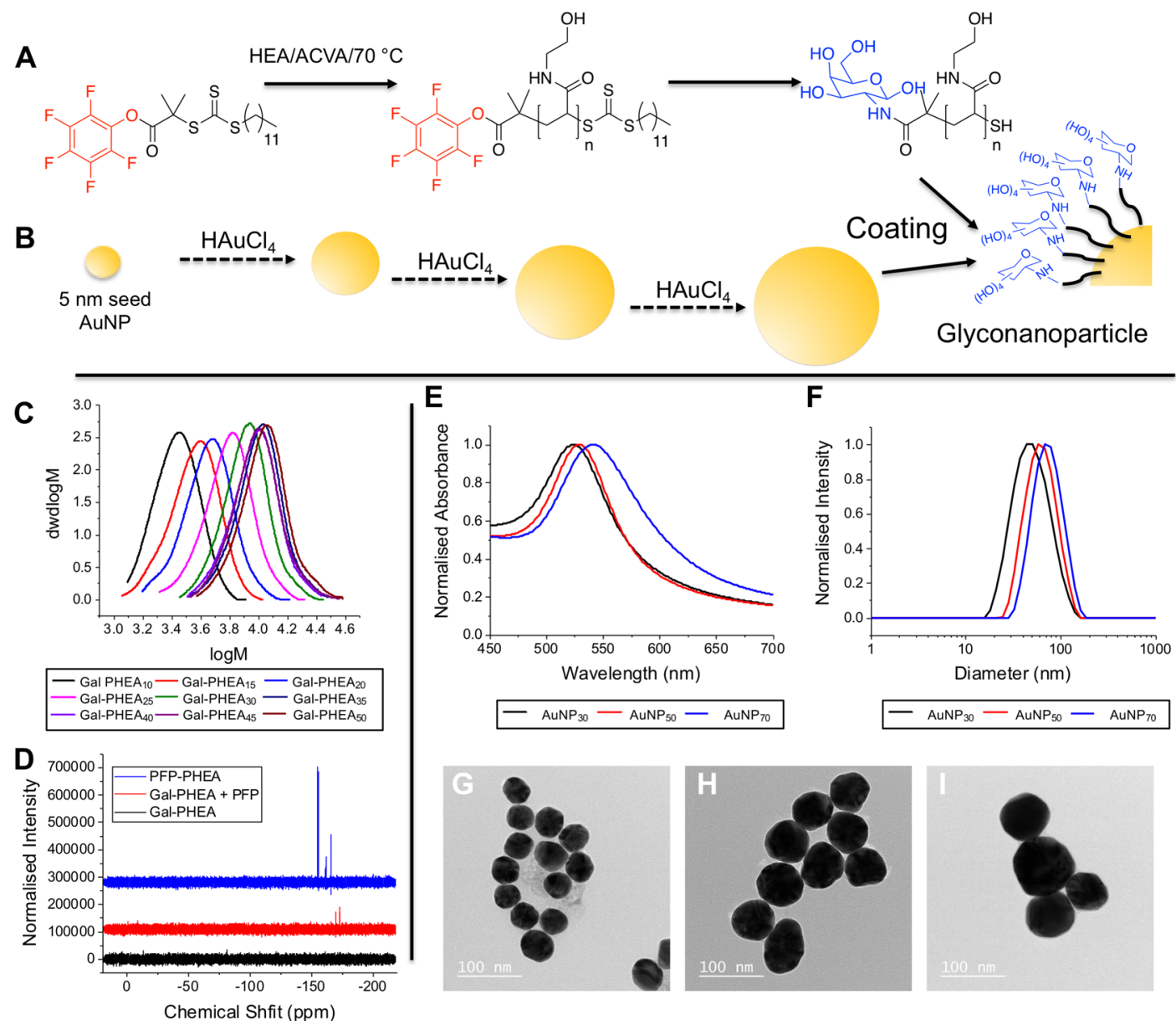

Figure 1. Synthetic strategy and characterization of polymers and particles. (A) Synthesis of galactose terminal PHEA; (B) step growth process for the synthesis of gold nanoparticles; (C) SEC of Gal-PHEAs; (D) ${ }^{19} \mathrm{~F}$ NMR showing displacement of the pentafluorophenyl unit; (E) UV-vis spectroscopy of uncoated AuNPs; (F) DLS of uncoated AuNPs; (G-I) TEM of uncoated AuNPs. Scale bar $=100 \mathrm{~nm}$.

consistent with polymer coating. X-ray photoelectron spectroscopy (XPS) was used to confirm polymer coating on AuNPs by increased N 1s (from amide) as well as C 1s signals (Supporting Information, Tables S1 and S2, Figures S2-S5).

To enable protein binding studies, it was essential to first screen the library for colloidal stability in the buffer to prevent false positives in aggregation screens. Both particle size and polymer length impact this, and it is a crucial step in any binding assay. The galactose functional particles were screened for aggregation stability by observing the increase in $\mathrm{Abs}_{700}$. A $\mathrm{NaCl}$ gradient $(0-1 \mathrm{M})$ as well as HEPES buffer were tested (full data set is in the Supporting Information, Figure S6). From this, it was clear that for all particle sizes, ligands with a DP above 25 were essential to obtain colloidally stable particles, in line with the previous reports. ${ }^{28,42}$ From this screen, it was decided to study protein binding using particles coated with polymers DP25-DP50 to avoid false positives due to buffer-induced aggregation. To screen for binding, the model lectin, SBA was used to validate the methods and to establish which particle structural requirements gave the strongest outputs, Figure 2.

Figure $2 \mathrm{~A}$ shows the outputs of this aggregation screen as a function of polymer ligand length and nanoparticle size. Full dose-dependent curves are in the Supporting Information (Figure S6). For all polymer lengths, the largest changes were seen for the shortest polymer chain length (DP25) with a stepwise decrease as polymer length increases, and the biggest (70 $\mathrm{nm}$ ) particles gave the largest outputs. Although the aggregation assay used above provides a convenient output of binding, it cannot rule out the case where lectins bind but fail to agglutinate. For example, the longer polymer linkers are likely able to bind to SBA, but also provide a steric block against agglutination, highlighting the need for tuning of these parameters. Therefore, a second assay using BLI was developed. $^{43}$ In short, biotinylated SBA was immobilized onto SA functional BLI sensors (ForteBio) (Figure 2B) and the nanoparticles exposed to this. As BLI is mass sensitive, individual glycans do not give a strong signal, but when coupled in this nanoparticle format, larger signals are obtained. In addition, cluster glycoside enhancement is achieved and is a key benefit of this approach. The total BLI signal as a function of particle parameters is shown in Figure 2D. The shorter polymers at all particle sizes gave the largest outputs; however, there was little binding observed to the SBA when DP50 polymers were used. This suggests that the lower density of longer polymers is reducing overall affinity and multivalent enhancement. Brewer and co-workers, ${ }^{44,45}$ and Godula and Bertozzi $^{46}$ observed valency-dependent binding of SBA to GalNAc-functionalized mucins/mucin-like materials. Furthermore, Gibson and co-workers have previously shown densitydependent binding of SBA to heterogenous AuNPs. ${ }^{31}$ Larger particles gave more signal, but it should be noted that BLI is a mass-weighted analysis method and hence only qualitative trends can be compared for nanoparticle size. 


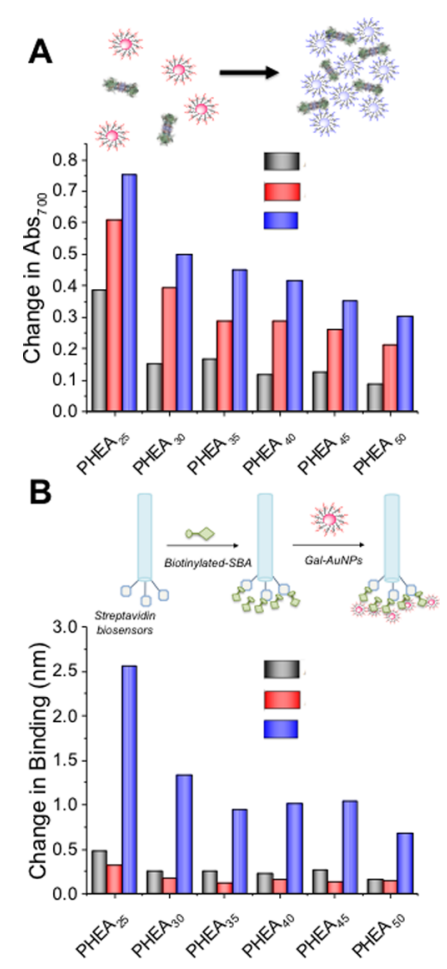

Figure 2. Galactosylated particles and SBA binding. (A) Aggregation of gold particles leading to red-blue color shift due to SPR band coupling. Change in $\mathrm{Abs}_{700}$ using $500 \mathrm{nM} \mathrm{SBA}$ as a function of coating and core size. Full binding curves from 500 to $0 \mathrm{nM}$ are in the Supporting Information, Figure S7; (B) BLI schematic, showing capture of SBA and subsequent binding to AuNPs to generate signal. Total binding $\left(\Delta_{\max }\right)$ as a function of coating and core size. Full binding curves are in the Supporting Information, Figure S8.

Guided by the above data, using galactose functional nanoparticles, the primary aim of studying multivalent sialic acid particles could be investigated on the optimized scaffold. 1-Deoxy, 1-amino, 2,3- and 2,6-sialyllactose were synthesized by the single-step Kochetkov amination method ${ }^{47}$ (Figure 3A, with the amination confirmed by mass spectrometry). Using the same procedure as $\mathrm{GalNH}_{2}$, the sialyllactosamines (SLs) were conjugated to DP25 PHEA and subsequently used to coat $70 \mathrm{~nm}$ AuNPs. This single polymer/particle combination was chosen based on the above screening (Figure 2), which ensured that particles that generate the largest signals with consistent grafting densities were used.

These glycoparticles were first tested against WGA, a lectin known to have some affinity to sialic acids. ${ }^{48}$ Using UV-vis to monitor aggregation, it appeared that both 2,3- and 2,6sialyllactosamines had similar affinities toward WGA (Figure $4 \mathrm{~A})$, which was not expected. However, parallel DLS investigations (Figure 4B) showed 2,6-sialyllactosamine to cluster faster and to give larger cluster sizes, which is consistent with it having a stronger interaction than 2,3-sialyllactosamine. BLI analysis against WGA immobilized on a surface showed greater specificity for the 2,6-sialyllactosamine compared to 2,3-sialyllactosamine, which is in line with the previous reports. This set of analyses shows that the colorimetric changes of glyconanoparticles are useful for comparisons of affinity, but more detailed methods (such as the BLI) are required, especially if unknown/new interactions are being evaluated.

The above data shows that DP25 PHEA ligands on $70 \mathrm{~nm}$ particles give strong binding, and that the amidation/ conjugation methodology enables installation of sialic acid isomers with retention of their biological binding specificities and affinities. The particles were then taken forward into a screen against a panel of HAs. A single nanoparticle/polymer length combination was used to ensure that grafting density differences did not affect the observed outcomes, where glycan density display is likely to play a significant role.

Influenza A can be categorized by the different subtypes of the proteins present of which there are at least 18 different HA subtypes named $\mathrm{H} 1$ to $\mathrm{H} 18 .^{2}$ The panel of HAs used were from the five strains with human hosts: $\mathrm{H} 1$ from A/California/ 07/2009 (H1N1)pdm09, H2 from A/Singapore/1/1957 (H2N2), H3 from A/Uruguay/716/2007 (H3N2), H7 from,

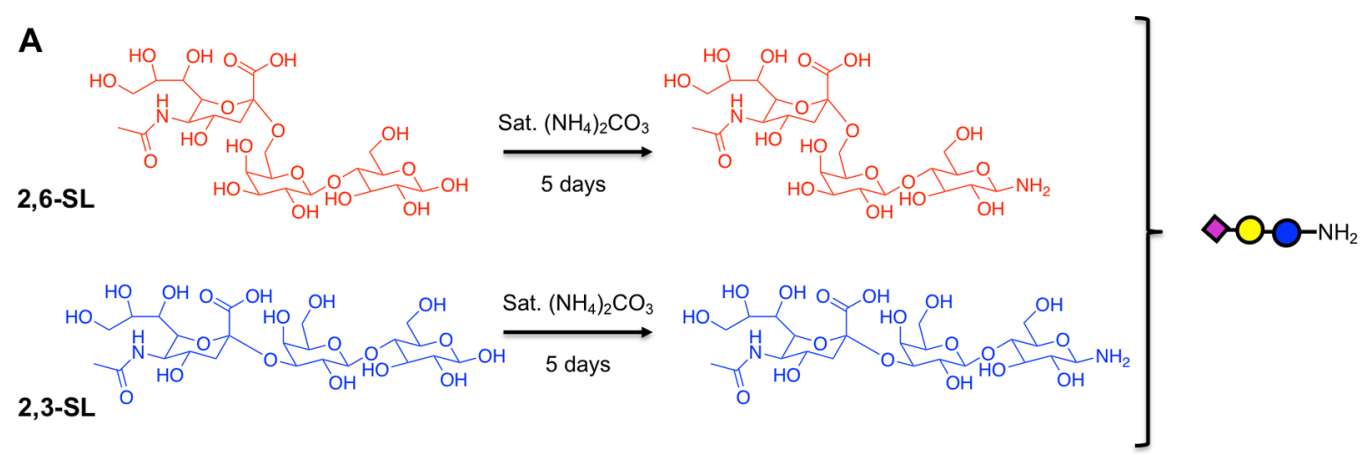

B
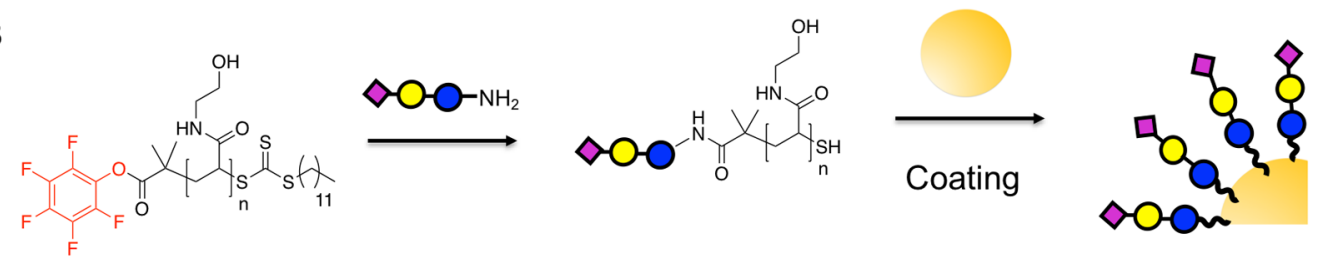

Glyconanoparticle

Figure 3. Synthesis of sialylated gold nanoparticles. (A) Amination of 2,3- and 2,6- sialyllactoses using Kochetkov method; (B) reaction of DP25 PFP-PHEA with sialyllactoamines and subsequent immobilization of the polymers onto $70 \mathrm{~nm}$ gold nanoparticles. 


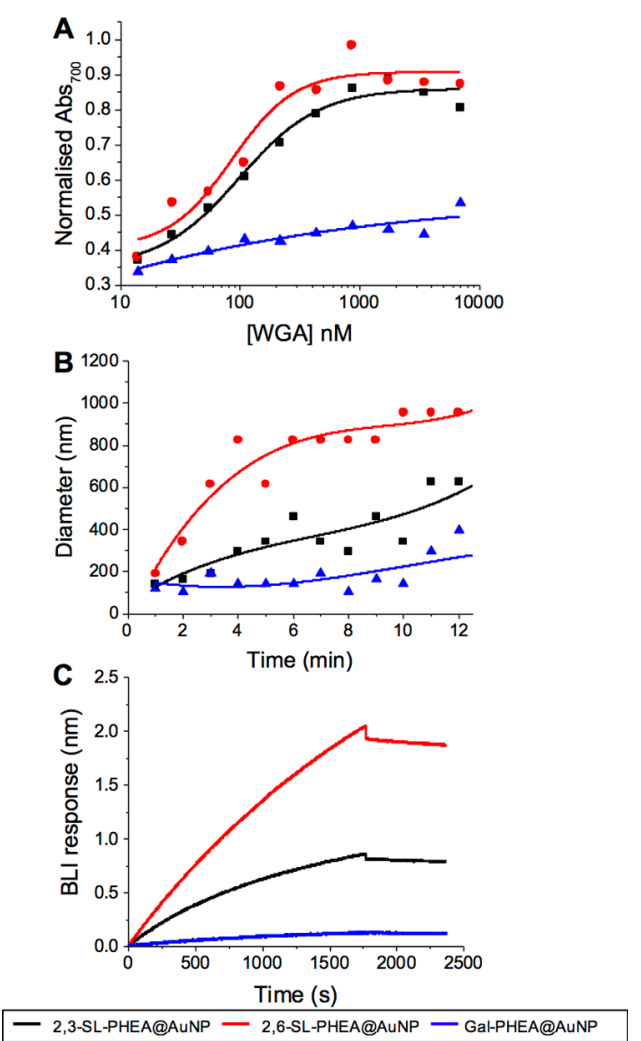

Figure 4. Interaction of SL-functionalized particles: 2,3-SL-PHEA@ AuNP (black - ), 2,6-SL-PHEA@AuNP (red - ), and Gal-PHEA@ AuNP (blue $\mathbf{\Delta}$ ) with WGA by (A) UV-vis; (B) DLS; and (C) BLI.

A/Canada/rv444/2004 (H7N3), and H9 from A/Hong Kong/33982/2009 (H9N2); and two from avian hosts: H4 from A/mallard/Alberta/455/2015 (H4N6) and H5 from A/ duck/Hunan/795/2002 (H5N1); and another zoonotic strain, H10 from A/harbor seal/Germany/1/2014 (H10N7) (seal host), and it enables the study of pathogenic processes without the need for intact viral particles. HAs were immobilized onto the BLI sensors using biotin/SA (as above). BLI has been widely used to evaluate hemagglutinin/neuraminidase function using isolated proteins and the whole virus. ${ }^{49-51}$ Figure 5A shows example BLI curves of 2,6-sialyllactosamine nanoparticles against the HA panel and Figure 5B shows BLI curves of 2,3-sialyllactosamine nanoparticles. There was a difference in response in terms of total mass captured and the rate of binding, reiterating the range of binding affinities shown by HAs from different pathogenic strains (low pathogenic avian strains) when presented in a multivalent format. To enable comparison, Figure 5C shows the maximal binding of the particles to the HAs for both 2,3- and 2,6-sialyllactosaminefunctionalized AuNPs. A key feature here is that although many of the avian HAs are characterized as being $\alpha 2,3-$ selective based on previous studies using monovalent compounds, there is clearly cross-affinity in most cases using our multivalent system. This shows the importance of this study, as it shows that glycan specificity can change when in multivalent format because of the enhancement of weak interactions, as well as stronger ones. $\mathrm{H} 9$ in particular showed significant binding to both 2,3-sialyllactosamine and 2,6sialyllactosamine AuNPs, indicating that it can bind to both human and avian sialic acids, which is consistent with the virus being of avian origins infecting a human host. This crossover in

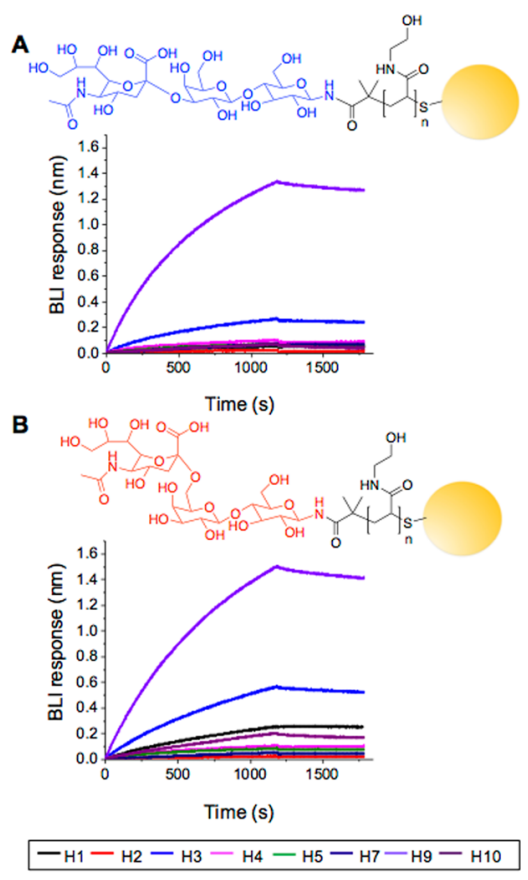

C
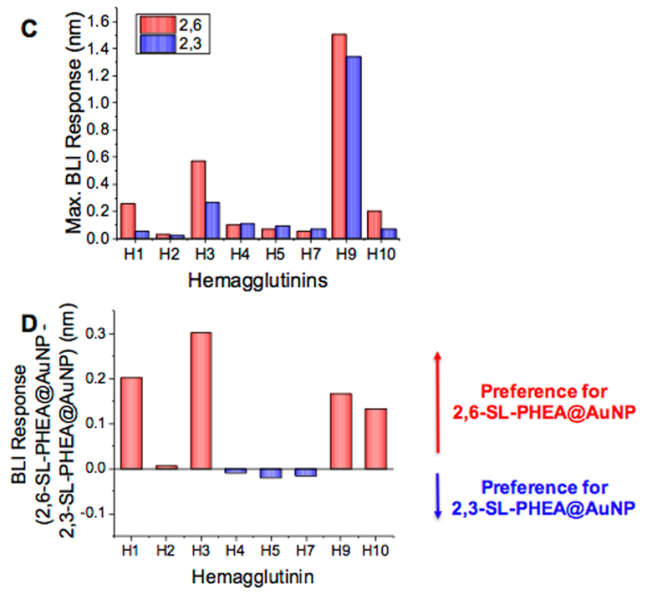

Figure 5. Interaction of SL-functionalized nanoparticles with a panel of HAs using BLI. (A) 2,3-SL-PHEA@AuNP and (B) 2,6-SLPHEA@AuNP BLI binding curves to HA panel ( $\mathrm{H} 1$ from A/ California/07/2009 (H1N1)pdm09, H2 from A/Singapore/1/1957 (H2N2), H3 from A/Uruguay/716/2007 (H3N2), H4 from A/ mallard/Alberta/455/2015 (H4N6), H5 from A/duck/Hunan/795/ 2002 (H5N1), H7 from, A/Canada/rv444/2004 (H7N3), NR-43740, H9 from A/Hong Kong/33982/2009 (H9N2), and H10 from A/ harbor seal/Germany/1/2014 (H10N7)); (C) comparison of the maximal binding of 2,3-SL-PHEA@AuNP and 2,6-SL-PHEA@AuNP to the HA panel; (D) difference (subtraction) between maximal binding to 2,6-SL-PHEA@AuNP and 2,3-SL-PHEA@AuNP to visualize relative binding preferences.

binding has also been observed by Godula and co-workers, ${ }^{24}$ where significant binding of H1N1 (A/Puerto Rico/8/34 which transmitted into humans) was noted toward both $\alpha 2,6$ and $\alpha 2,3$ sialylated polymer microarrays. In contrast, more discrete binding was observed with H3N2 (A/Aichi/2/68) which bound only to $\alpha 2,6$ but not to $\alpha 2,3$ sialylated polymer arrays (note that the specific strains were not the same as used in this study). Furthermore, McCauley and co-workers have investigated the mammalian transmissibility of many avian and other zoonotic influenza strains including H7N9, ${ }^{49} \mathrm{H} 9 \mathrm{~N} 2,{ }^{50}$ 
and $\mathrm{H} 10 \mathrm{~N} 8^{52}$ by looking at the binding of sialylated polymers to viruses. In particular, a panel of $\mathrm{H} 9 \mathrm{~N} 2$ viruses from different origins were assessed for their binding to $\alpha 2,6$ and $\alpha 2,3$ sialylated polymer-functionalized BLI sensors. ${ }^{50}$ Wide-ranging binding variability was noted to different $\mathrm{H} 9 \mathrm{~N} 2$ viruses, with the H9N2 (A/Hong Kong/33982/2009) virus (the same H9 used in this study) showing greater binding toward $\alpha 2,6$ sialylated polymers over $\alpha 2,3$ sialylated polymers, which is in agreement with our results here, supporting the approach taken (dose-dependent BLI binding curves for $\mathrm{H} 1$ from $\mathrm{A} /$ California/07/2009 (H1N1)pdm09, H7 from A/Canada/ rv444/2004 (H7N3), and H9 from H9N2 (A/Hong Kong/ $33982 / 2009$ ) in the Supporting Information, Figures S12S14). The H7N9 strain has been reported to retain its avian affinity preference even with mutations to target human receptors, which we also observed using this nanoparticle system. ${ }^{53}$ To visualize these differences, Figure $5 \mathrm{D}$ plots the relative binding preferences, defined as the maximal binding of 2,6-sialyllactosamine nanoparticles, minus the maximal binding of 2,3-sialyllactosamine to each hemagglutinin. As the same coating and same nanoparticle core was used in each case, this enabled visualization of the differences in total particle captured to the haemagglutinin. These results show that our nanoparticle system generates sufficient signal amplification to enable the evaluation of glycans for binding haemagglutinins and to unravel the cross-affinity due to the multivalent presentation. It is important to note that these results may be specific to the sialyllactose ligands being employed here, which are readily available compared to, for example, complex branched glycans. It does show that, however, in multivalent format both the extent of binding and relative binding between the isomers varies between the hemagglutinins and may offer routes to multiplexed sensing and discrimination.

\section{CONCLUSIONS}

Here, we have developed a multivalent nanoparticle approach to investigate the affinity of sialic acid isomers against a panel of influenza A HAs to map their affinity. This allows for the probing of specificity and affinity trends while benefitting from signal amplification due to particle size. The multivalent nanoparticles were synthesized using polymeric ligands bearing a glycan capture (pentafluorophenyl ester) and a gold immobilization (thiol) termini. Initial screening of a library of 27 nanoparticles, varying in ligand length and core diameter, enabled identification of the optimum multivalent probe, using a model lectin and complementary UV-vis, DLS, and BLI analysis. 2,3- and 2,6-sialyllactosamine were conjugated to these particles via a Kochetkov amination step, which were then interrogated for their binding against a panel of influenza A HAs including three mammalian strains, four avian strains, and a zoonotic influenza strain. As expected, 2,3-sialyllactosamine had strong affinity toward avian strains and 2,6sialyllactosamine against mammalian. However, the analysis also revealed that in multivalent format, there was significant crossover in affinities. All avian influenzas showed affinity toward the multivalent 2,6-sialyllactosamine particles suggesting the potential for crossover due to multivalency. It is important to note that this study does not predict zoonosis potential, but rather highlights that multivalent nanoparticle presentation affects the affinity/selectivity trends compared to monovalent compounds. The identification of off-specific binding profiles is crucial to help develop nanomaterials for biosensing and monitoring of emerging infectious diseases.

\section{ASSOCIATED CONTENT}

\section{Supporting Information}

The Supporting Information is available free of charge at https://pubs.acs.org/doi/10.1021/acs.biomac.0c00179.

Additional nanoparticle characterization (XPS and DLS), full UV-visible spectroscopy curves for the lectin binding assays, and underpinning BLI binding curves (PDF)

\section{AUTHOR INFORMATION}

\section{Corresponding Author}

Matthew I. Gibson - Department of Chemistry and Warwick Medical School, University of Warwick, Coventry CV4 7AL, U.K.; $\odot$ orcid.org/0000-0002-8297-1278;

Email: m.i.gibson@warwick.ac.uk

\section{Authors}

Sarah-Jane Richards - Department of Chemistry, University of Warwick, Coventry CV4 7AL, U.K.

Alexander N. Baker - Department of Chemistry, University of Warwick, Coventry CV4 7AL, U.K.; 10 orcid.org/0000-00016019-3412

Marc Walker - Department of Physics, University of Warwick, Coventry CV4 7AL, U.K.

Complete contact information is available at:

https://pubs.acs.org/10.1021/acs.biomac.0c00179

\section{Notes}

The authors declare no competing financial interest. The research data supporting this publication can be found at http://wrap.warwick.ac.uk.

\section{ACKNOWLEDGMENTS}

M.I.G. holds an ERC starting grant (CRYOMAT 638661). The Royal Society are also thanked for funding the cryomicroscopes used in this study. BBSRC/Innovate are thanked for funding the Specialty Glycans project BB/M02878X/1. The BBSRC-funded MIBTP program (BB/M01116X/1) and Iceni Diagnostics ltd are thanked for a studentship for A.N.B. The Warwick Polymer Research Technology Platform is acknowledged for the SEC analysis. Laura Wilkins (UoW) is thanked for the TEM analysis. The following reagents were obtained through BEI Resources, NIAID, NIH: H1 HA protein from influenza virus, A/California/07/2009 (H1N1) pdm09, recombinant from baculovirus, NR-44074, H2 HA protein from influenza virus, A/Singapore/1/1957 (H2N2), recombinant from baculovirus, NR-2668, H3 HA protein from influenza virus, A/Uruguay/716/2007 (H3N2), recombinant from baculovirus, NR-15168, H4 HA protein from influenza virus, A/mallard/Alberta/455/2015 (H4N6), recombinant from baculovirus, NR-51128, H5 HA protein from influenza virus, A/duck/Hunan/795/2002 (H5N1), recombinant from baculovirus, NR-43739, H7 HA protein from influenza virus, A/Canada/rv444/2004 (H7N3), recombinant from baculovirus, NR-43740, H9 HA protein from influenza virus, A/Hong Kong/33982/2009 (H9N2), recombinant from baculovirus, NR-41792 and H10 HA protein with C-terminal histidine tag from influenza virus, A/harbor seal/Germany/1/2014 (H10N7), recombinant from baculovirus, NR-50172. 


\section{REFERENCES}

(1) Iuliano, A. D.; Roguski, K. M.; Chang, H. H.; Muscatello, D. J.; Palekar, R.; Tempia, S.; Cohen, C.; Gran, J. M.; Schanzer, D.; Cowling, B. J.; Wu, P.; Kyncl, J.; Ang, L. W.; Park, M.; RedlbergerFritz, M.; Yu, H.; Espenhain, L.; Krishnan, A.; Emukule, G.; van Asten, L.; Pereira da Silva, S.; Aungkulanon, S.; Buchholz, U.; Widdowson, M.-A.; Bresee, J. S.; Azziz-Baumgartner, E.; Cheng, P.-Y.; Dawood, F.; Foppa, I.; Olsen, S.; Haber, M.; Jeffers, C.; MacIntyre, C. R.; Newall, A. T.; Wood, J. G.; Kundi, M.; Popow-Kraupp, T.; Ahmed, M.; Rahman, M.; Marinho, F.; Sotomayor Proschle, C. V.; Vergara Mallegas, N.; Luzhao, F.; Sa, L.; Barbosa-Ramírez, J.; Sanchez, D. M.; Gomez, L. A.; Vargas, X. B.; Acosta Herrera, a.; Llanés, M. J.; Fischer, T. K.; Krause, T. G.; Mølbak, K.; Nielsen, J.; Trebbien, R.; Bruno, A.; Ojeda, J.; Ramos, H.; an der Heiden, M.; del Carmen Castillo Signor, L.; Serrano, C. E.; Bhardwaj, R.; Chadha, M.; Narayan, V.; Kosen, S.; Bromberg, M.; Glatman-Freedman, A.; Kaufman, Z.; Arima, Y.; Oishi, K.; Chaves, S.; Nyawanda, B.; AlJarallah, R. A.; Kuri-Morales, P. A.; Matus, C. R.; Corona, M. E. J.; Burmaa, A.; Darmaa, O.; Obtel, M.; Cherkaoui, I.; van den Wijngaard, C. C.; van der Hoek, W.; Baker, M.; Bandaranayake, D.; Bissielo, A.; Huang, S.; Lopez, L.; Newbern, C.; Flem, E.; Grøneng, G. M.; Hauge, S.; de Cosío, F. G.; de Moltó, Y.; Castillo, L. M.; Cabello, M. A.; von Horoch, M.; Medina Osis, J.; Machado, A.; Nunes, B.; Rodrigues, A. P.; Rodrigues, E.; Calomfirescu, C.; Lupulescu, E.; Popescu, R.; Popovici, O.; Bogdanovic, D.; Kostic, M.; Lazarevic, K.; Milosevic, Z.; Tiodorovic, B.; Chen, M.; Cutter, J.; Lee, V.; Lin, R.; Ma, S.; Cohen, A. L.; Treurnicht, F.; Kim, W. J.; Delgado-Sanz, C.; de mateo Ontañón, S.; Larrauri, A.; León, I. L.; Vallejo, F.; Born, R.; Junker, C.; Koch, D.; Chuang, J.-H.; Huang, W.-T.; Kuo, H.-W.; Tsai, Y.-C.; Bundhamcharoen, K.; Chittaganpitch, M.; Green, H. K.; Pebody, R.; Goñi, N.; Chiparelli, H.; Brammer, L.; Mustaquim, D. Estimates of Global Seasonal Influenza-Associated Respiratory Mortality: A Modelling Study. Lancet 2018, 391, 1285-1300.

(2) World Heath Organisation. Fact Sheet-Influenza (Seasonal). https://www.who.int/news-room/fact-sheets/detail/influenza(seasonal) (accessed March 10, 2020).

(3) Rogers, G. N.; D'Souza, B. L. Receptor Binding Properties of Human and Animal H1 Influenza Virus Isolates. Virology 1989, 173, 317-322.

(4) Connor, R. J.; Kawaoka, Y.; Webster, R. G.; Paulson, J. C. Receptor Specificity in Human, Avian, and Equine $\mathrm{H} 2$ and $\mathrm{H} 3$ Influenza Virus Isolates. Virology 1994, 205, 17-23.

(5) Webster, R. G.; Laver, W. G.; Air, G. M.; Schild, G. C. Molecular Mechanisms of Variation in Influenza Viruses. Nature 1982, 296, $115-121$.

(6) Gamblin, S. J.; Haire, L. F.; Russell, R. J.; Stevens, D. J.; Xiao, B.; Ha, Y.; Vasisht, N.; Steinhauer, D. A.; Daniels, R. S.; Elliot, A.; Wiley, D. C.; Skehel, J. J. The Structure and Receptor Binding Properties of the 1918 Influenza Hemagglutinin. Science 2004, 303, 1838-1842.

(7) Rogers, G. N.; Paulson, J. C.; Daniels, R. S.; Skehel, J. J.; Wilson, I. A.; Wiley, D. C. Single Amino Acid Substitutions in Influenza Haemagglutinin Change Receptor Binding Specificity. Nature 1983, 304, 76-78.

(8) World Health Organisation. Fact Sheet-Influenza (Avian and other zoonotic). https://www.who.int/news-room/fact-sheets/detail/ influenza-(avian-and-other-zoonotic) (accessed March 10, 2020).

(9) Broszeit, F.; Tzarum, N.; Zhu, X.; Nemanichvili, N.; Eggink, D.; Leenders, T.; Li, Z.; Liu, L.; Wolfert, M. A.; Papanikolaou, A.; Martínez-Romero, C.; Gagarinov, I. A.; Yu, W.; García-Sastre, A.; Wennekes, T.; Okamatsu, M.; Verheije, M. H.; Wilson, I. A.; Boons, G.-J.; de Vries, R. P. N-Glycolylneuraminic Acid as a Receptor for Influenza A Viruses. Cell Rep. 2019, 27, 3284-3294.e6.

(10) Byrd-Leotis, L.; Jia, N.; Dutta, S.; Trost, J. F.; Gao, C.; Cummings, S. F.; Braulke, T.; Müller-Loennies, S.; HeimburgMolinaro, J.; Steinhauer, D. A.; Cummings, R. D. Influenza Binds Phosphorylated Glycans from Human Lung. Sci. Adv. 2019, 5, No. eaav2554.
(11) Gulati, S.; Lasanajak, Y.; Smith, D. F.; Cummings, R. D.; Air, G. M. Glycan Array Analysis of Influenza H1N1 Binding and Release. Cancer Biomarkers 2014, 14, 43-53.

(12) Sauter, N. K.; Bednarski, M. D.; Wurzburg, B. A.; Hanson, J. E.; Whitesides, G. M.; Skehel, J. J.; Wiley, D. C. Hemagglutinins from Two Influenza Virus Variants Bind to Sialic Acid Derivatives with Millimolar Dissociation Constants: A $500-\mathrm{MHz}$ Proton Nuclear Magnetic Resonance Study. Biochemistry 1989, 28, 8388-8396.

(13) Weis, W.; Brown, J. H.; Cusack, S.; Paulson, J. C.; Skehel, J. J.; Wiley, D. C. Structure of the Influenza Virus Haemagglutinin Complexed with Its Receptor, Sialic Acid. Nature 1988, 333, 426431.

(14) Lundquist, J. J.; Toone, E. J. The Cluster Glycoside Effect. Chem. Rev. 2002, 102, 555-578.

(15) Gou, Y.; Richards, S.-J.; Haddleton, D. M.; Gibson, M. I. Investigation of Glycopolymer-Lectin Interactions Using QCM-d: Comparison of Surface Binding with Inhibitory Activity. Polym. Chem. 2012, 3, 1634-1640.

(16) Gou, Y.; Geng, J.; Richards, S.-J.; Burns, J.; Remzi Becer, C.; Haddleton, D. M. A Detailed Study on Understanding Glycopolymer Library and Con A Interactions. J. Polym. Sci., Part A: Polym. Chem. 2013, 51, 2588-2597.

(17) Chen, Y.; Chen, G.; Stenzel, M. H. Synthesis and Lectin Recognition of Glyco Star Polymers Prepared by "Clicking" Thiocarbohydrates onto a Reactive Scaffold. Macromolecules 2010, 43, 8109-8114.

(18) Ponader, D.; Wojcik, F.; Beceren-Braun, F.; Dernedde, J.; Hartmann, L. Sequence-Defined Glycopolymer Segments Presenting Mannose: Synthesis and Lectin Binding Affinity. Biomacromolecules 2012, 13, 1845-1852.

(19) Baradel, N.; Fort, S.; Halila, S.; Badi, N.; Lutz, J.-F. Synthesis of Single-Chain Sugar Arrays. Angew. Chem., Int. Ed. 2013, 52, 23352339.

(20) Mammen, M.; Dahmann, G.; Whitesides, G. M. Effective Inhibitors of Hemagglutination by Influenza Virus Synthesized from Polymers Having Active Ester Groups. Insight into Mechanism of Inhibition. J. Med. Chem. 1995, 38, 4179-4190.

(21) Sigal, G. B.; Mammen, M.; Dahmann, G.; Whitesides, G. M. Polyacrylamides Bearing Pendant $\alpha$-Sialoside Groups Strongly Inhibit Agglutination of Erythrocytes by Influenza Virus: The Strong Inhibition Reflects Enhanced Binding through Cooperative Polyvalent Interactions. J. Am. Chem. Soc. 1996, 118, 3789-3800.

(22) Nagao, M.; Fujiwara, Y.; Matsubara, T.; Hoshino, Y.; Sato, T.; Miura, Y. Design of Glycopolymers Carrying Sialyl Oligosaccharides for Controlling the Interaction with the Influenza Virus. Biomacromolecules 2017, 18, 4385-4392.

(23) Lu, W.; Du, W.; Somovilla, V. J.; Yu, G.; Haksar, D.; de Vries, E.; Boons, G.-J.; de Vries, R. P.; de Haan, C. A. M.; Pieters, R. J. Enhanced Inhibition of Influenza A Virus Adhesion by Di- and Trivalent Hemagglutinin Inhibitors. J. Med. Chem. 2019, 62, 63986404.

(24) Huang, M. L.; Cohen, M.; Fisher, C. J.; Schooley, R. T.; Gagneux, P.; Godula, K. Determination of Receptor Specificities for Whole Influenza Viruses Using Multivalent Glycan Arrays. Chem. Commun. 2015, 51, 5326-5329.

(25) Bandlow, V.; Liese, S.; Lauster, D.; Ludwig, K.; Netz, R. R.; Herrmann, A.; Seitz, O. Spatial Screening of Hemagglutinin on Influenza A Virus Particles: Sialyl-LacNAc Displays on DNA and PEG Scaffolds Reveal the Requirements for Bivalency Enhanced Interactions with Weak Monovalent Binders. J. Am. Chem. Soc. 2017, 139, 16389-16397.

(26) Peng, W.; de Vries, R. P.; Grant, O. C.; Thompson, A. J.; McBride, R.; Tsogtbaatar, B.; Lee, P. S.; Razi, N.; Wilson, I. A.; Woods, R. J.; Paulson, J. C. Recent H3N2 Viruses Have Evolved Specificity for Extended, Branched Human-Type Receptors, Conferring Potential for Increased Avidity. Cell Host Microbe 2017, 21, 2334.

(27) Bhatia, S.; Lauster, D.; Bardua, M.; Ludwig, K.; AngiolettiUberti, S.; Popp, N.; Hoffmann, U.; Paulus, F.; Budt, M.; Stadtmüller, 
M.; Wolff, T.; Hamann, A.; Böttcher, C.; Herrmann, A.; Haag, R. Linear Polysialoside Outperforms Dendritic Analogs for Inhibition of Influenza Virus Infection in Vitro and in Vivo. Biomaterials 2017, 138, 22-34.

(28) Richards, S.-J.; Gibson, M. I. Optimization of the Polymer Coating for Glycosylated Gold Nanoparticle Biosensors to Ensure Stability and Rapid Optical Readouts. ACS Macro Lett. 2014, 3, 1004-1008.

(29) Richards, S.-J.; Otten, L.; Gibson, M. I.; Besra, G. S.; Gibson, M. I. Glycosylated Gold Nanoparticle Libraries for Label-Free Multiplexed Lectin Biosensing. J. Mater. Chem. B 2016, 4, 30463053.

(30) Richards, S.-J.; Biggs, C. I.; Gibson, M. I. Multivalent Glycopolymer-Coated Gold Nanoparticles. Macro-Glycoligands; Methods in Molecular Biology; Springer, 2016; Vol. 1367, pp 169-179.

(31) Otten, L.; Vlachou, D.; Richards, S.-J.; Gibson, M. I. Glycan Heterogeneity on Gold Nanoparticles Increases Lectin Discrimination Capacity in Label-Free Multiplexed Bioassays. Analyst 2016, 141, 4305-4312.

(32) Marín, M. J.; Rashid, A.; Rejzek, M.; Fairhurst, S. A.; Wharton, S. A.; Martin, S. R.; McCauley, J. W.; Wileman, T.; Field, R. A.; Russell, D. A. Glyconanoparticles for the Plasmonic Detection and Discrimination between Human and Avian Influenza Virus. Org. Biomol. Chem. 2013, 11, 7101.

(33) Poonthiyil, V.; Nagesh, P. T.; Husain, M.; Golovko, V. B.; Fairbanks, A. J. Gold Nanoparticles Decorated with Sialic Acid Terminated Bi-Antennary N-Glycans for the Detection of Influenza Virus at Nanomolar Concentrations. ChemistryOpen 2015, 4, 708716.

(34) Zhang, Z.; Schepens, B.; Nuhn, L.; Saelens, X.; Schotsaert, M.; Callewaert, N.; De Rycke, R.; Zhang, Q.; Moins, S.; Benali, S.; Mespouille, L.; Hoogenboom, R.; De Geest, B. G. Influenza-Binding Sialylated Polymer Coated Gold Nanoparticles Prepared via RAFT Polymerization and Reductive Amination. Chem. Commun. 2016, 52, 3352-3355.

(35) Papp, I.; Sieben, C.; Ludwig, K.; Roskamp, M.; Böttcher, C.; Schlecht, S.; Herrmann, A.; Haag, R. Inhibition of Influenza Virus Infection by Multivalent Sialic-Acid- Functionalized Gold Nanoparticles. Small 2010, 6, 2900-2906.

(36) Richards, S.-J.; Fullam, E.; Besra, G. S.; Gibson, M. I. Discrimination between Bacterial Phenotypes Using Glyco-Nanoparticles and the Impact of Polymer Coating on Detection Readouts. J. Mater. Chem. B 2014, 2, 1490-1498.

(37) Di Iorio, D.; Verheijden, M. L.; Van Der Vries, E.; Jonkheijm, P.; Huskens, J. Weak Multivalent Binding of Influenza Hemagglutinin Nanoparticles at a Sialoglycan-Functionalized Supported Lipid Bilayer. ACS Nano 2019, 13, 3413-3423.

(38) Kwon, S.-J.; Na, D. H.; Kwak, J. H.; Douaisi, M.; Zhang, F.; Park, E. J.; Park, J.-H.; Youn, H.; Song, C.-S.; Kane, R. S.; Dordick, J. S.; Lee, K. B.; Linhardt, R. J. Nanostructured Glycan Architecture Is Important in the Inhibition of Influenza A Virus Infection. Nat. Nanotechnol. 2017, 12, 48-54.

(39) Bastús, N. G.; Comenge, J.; Puntes, V. Kinetically Controlled Seeded Growth Synthesis of Citrate-Stabilized Gold Nanoparticles of up to $200 \mathrm{Nm}$ : Size Focusing versus Ostwald Ripening. Langmuir 2011, 27, 11098-11105.

(40) Richards, S.-J.; Otten, L.; Gibson, M. I. Glycosylated Gold Nanoparticle Libraries for Label-Free Multiplexed Lectin Biosensing. J. Mater. Chem. B 2016, 4, 3046-3053.

(41) Haiss, W.; Thanh, N. T. K.; Aveyard, J.; Fernig, D. G. Determination of Size and Concentration of Gold Nanoparticles from UV-Vis Spectra. Anal. Chem. 2007, 79, 4215-4221.

(42) Won, S.; Richards, S.-J.; Walker, M.; Gibson, M. I. Externally Controllable Glycan Presentation on Nanoparticle Surfaces to Modulate Lectin Recognition. Nanoscale Horiz. 2017, 2, 106-109.

(43) Petersen, R. L. Strategies Using Bio-Layer Interferometry Biosensor Technology for Vaccine Research and Development. Biosensors 2017, 7, 49.
(44) Dam, T. K.; Gerken, T. A.; Brewer, C. F. Thermodynamics of Multivalent Carbohydrate-Lectin Cross-Linking Interactions: Importance of Entropy in the Bind and Jump Mechanism. Biochemistry 2009, 48, 3822-3827.

(45) Dam, T. K.; Gerken, T. A.; Cavada, B. S.; Nascimento, K. S.; Moura, T. R.; Brewer, C. F. Binding Studies of Alpha-GalNAc-Specific Lectins to the Alpha-GalNAc (Tn-Antigen) Form of Porcine Submaxillary Mucin and Its Smaller Fragments. J. Biol. Chem. 2007, 282, 28256-28263.

(46) Godula, K.; Bertozzi, C. R. Density Variant Glycan Microarray for Evaluating Cross-Linking of Mucin-like Glycoconjugates by Lectins. J. Am. Chem. Soc. 2012, 134, 15732-15742.

(47) Hermanson, G. T. Functional Targets. Bioconjugate Techniques, 3rd ed.; Academic Press: London, 2013; Chapter 1.

(48) Peters, B. P.; Ebisu, S.; Goldstein, I. J.; Flashner, M. Interaction of Wheat Germ Agglutinin with Sialic Acid. Biochemistry 1979, 18, $5505-5511$.

(49) Benton, D. J.; Martin, S. R.; Wharton, S. A.; McCauley, J. W. Biophysical Measurement of the Balance of Influenza A Hemagglutinin and Neuraminidase Activities. J. Biol. Chem. 2015, 290, 6516-6521.

(50) Peacock, T. P.; Benton, D. J.; Sadeyen, J. R.; Chang, P.; Sealy, J. E.; Bryant, J. E.; Martin, S. R.; Shelton, H.; McCauley, J. W.; Barclay, W. S.; Iqbal, M. Variability in H9N2 Haemagglutinin ReceptorBinding Preference and the $\mathrm{PH}$ of Fusion. Emerging Microbes Infect. 2017, 6, 1-7.

(51) Du, W.; Guo, H.; Nijman, V. S.; Doedt, J.; van der Vries, E.; van der Lee, J.; Li, Z.; Boons, G.-J.; van Kuppeveld, F. J. M.; de Vries, E.; Matrosovich, M.; de Haan, C. A. M. The 2nd Sialic Acid-Binding Site of Influenza A Virus Neuraminidase Is an Important Determinant of the Hemagglutinin-Neuraminidase-Receptor Balance. PLoS Pathog. 2019, 15, No. e1007860.

(52) Vachieri, S. G.; Xiong, X.; Collins, P. J.; Walker, P. A.; Martin, S. R.; Haire, L. F.; Zhang, Y.; McCauley, J. W.; Gamblin, S. J.; Skehel, J. J. Receptor Binding by H10 Influenza Viruses. Nature 2014, 511, $475-477$.

(53) Xiong, X.; Martin, S. R.; Haire, L. F.; Wharton, S. A.; Daniels, R. S.; Bennett, M. S.; McCauley, J. W.; Collins, P. J.; Walker, P. A.; Skehel, J. J.; Gamblin, S. J. Receptor Binding by an H7N9 Influenza Virus from Humans. Nature 2013, 499, 496-499. 